\title{
Analysis of Polarization Splitters Composed of Multilayer Thin-Film Waveguides Using the Beam-Propagation Method Based on Yee's Mesh
}

\author{
Junji Yamauchi, Member, IEEE, Member, OSA, Yuji Kamei, Koichi Murase, and Hisamatsu Nakano, Fellow, IEEE
}

\begin{abstract}
Multilayer thin-film waveguides (MTFWs) are analyzed using the beam-propagation method based on Yee's mesh. The birefringence properties of the MTFW are demonstrated by the eigenmode analysis based on the imaginary-distance procedure. Taking advantage of the birefringence, a polarization splitter composed of two parallel MTFWs is proposed. Calculation shows that a short device length of $270 \mu \mathrm{m}$ is obtainable with crosstalks of $-33.5 \mathrm{~dB}$ for the quasi-transverse-electric mode and $-20.7 \mathrm{~dB}$ for the quasi-transverse-magnetic mode. A crossing-type polarization splitter is also developed, and the device length is further reduced to $120 \mu \mathrm{m}$.
\end{abstract}

Index Terms-Beam-propagation method, eigenmode analysis, multilayer thin-film waveguide (MTFW), polarization splitter, Yee's mesh.

\section{INTRODUCTION}

I $\mathrm{T}$ IS well-known that a multilayer thin-film waveguide (MTFW) has form birefringence and produces a large mode separation between the quasi-transverse-electric (TE) $E^{x}$ mode and the quasi-transverse-magnetic (TM) $E^{y}$ mode [1]-[4]. For this reason, the use of the MTFW makes it possible to realize a polarization-maintaining property. The MTFWs are composed of alternate thin layers of two isotropic materials with different refractive indexes. So far, various kinds of devices that are composed of the MTFW have been investigated [5], [6]. However, little attention has been paid to the 3-D propagating beam analysis because of its structural complexity.

For the 3-D propagating beam analysis, it is necessary to analyze the eigenmode field for the incident field. To date, many methods have been proposed for the eigenmode analysis [7]-[10]. One of them is an imaginary-distance propagation method [8] based on Yee's mesh (YM-BPM) [10]-[13]. The YM-BPM has the advantage that all electric- and magnetic-field components are simultaneously evaluated, and the obtained eigenmode field is directly utilized for the real-axis YM-BPM [13] and the finite-difference time-domain method [14].

In this paper, the eigenmodes of MTFWs are analyzed using the imaginary-distance YM-BPM [10]. The effective indexes as

Manuscript received January 15, 2007; revised April 13, 2007. This work was supported in part by the "University-Industry Joint Research" Project for Private Universities matching fund subsidy from the Ministry of Education, Culture, Sports, Science, and Technology (2003-2007).

J. Yamauchi and H. Nakano are with the Faculty of Engineering, Hosei University, Tokyo 184-8584, Japan.

Y. Kamei is with Topcon Corporation, Tokyo 174-8580, Japan.

$\mathrm{K}$. Murase is with Tokyo Electric Power Company, Tokyo 100-8560, Japan.

Digital Object Identifier 10.1109/JLT.2007.901376 a function of waveguide width are investigated. The evaluated effective indexes are in good agreement with previously published data [4].

As an application of the MTFW, a rib-type MTFW coupler is treated. This MTFW is a polarization-maintaining waveguide, i.e., the $E^{y}$ modes are cut off. The coupling length as a function of spacing between the waveguides is calculated. The results are compared with those of the single-layer rib-type waveguide coupler whose refractive index of the guiding layer is determined from the weighted average of the indexes of the MTFW. It is shown that the coupling lengths of the MTFW nearly agree with those of the single-layer waveguide for the $E_{11}^{x}$ mode.

Next, we propose a directional-coupler-type polarization splitter that consists of two parallel MTFWs [15]. By the 3-D eigenmode and propagating beam analyses, the propagation characteristics of the polarization splitter are investigated. For the eigenmode analysis, the coupling lengths of the quasi-TE and quasi-TM modes are evaluated. For the propagating beam analysis, the crosstalks are calculated. The analysis is carried out using the real- and imaginary-distance YM-BPMs [10], [13]. Numerical results show that the crosstalks are $-33.5 \mathrm{~dB}$ for the quasi-TE mode and $-20.7 \mathrm{~dB}$ for the quasi-TM mode.

We then compare the present polarization splitter with a splitter using a single MTFW (a similar splitter using a single MTFW was proposed in [6]). It is found that the splitter consisting of the two parallel MTFWs has the advantage that a short device length of $270 \mu \mathrm{m}$ is available.

Finally, we study a splitter that is composed of crossing MTFWs [16]. The device length is further reduced to $120 \mu \mathrm{m}$, with the crosstalk being about $-19 \mathrm{~dB}$ for the quasi-TE and quasi-TM modes.

\section{Numerical Method}

\section{A. Eigenmode Analysis}

The imaginary-distance YM-BPM [10]-[12] is employed to analyze the eigenmodes of MTFWs. This method that was proposed by Lee [10] is the full-vectorial explicit finite-difference method based on Yee's mesh.

In the imaginary-distance procedure [8], an arbitrary input field converts into the lowest eigenmode field, as the input field propagates in the imaginary-axis direction. For calculating higher order modes, we employ a Gram-Schmidt orthogonalization technique [10], [13], [17]. 


\section{B. Propagating Beam Analysis}

The explicit YM-BPM has the disadvantage that it cannot be used in the propagating beam (real-axis) analysis. Recently, the implicit YM-BPM [13] has been developed to resolve this problem. We adopt this method to calculate the propagating beam of polarization splitters using MTFWs. For the polarization splitters that are considered in this paper, it is confirmed from preliminary calculations that the results that were obtained from the semivectorial YM-BPM agree well with those from the full-vectorial YM-BPM. Since the computational efficiency of the semivectorial YM-BPM is higher than that of the fullvectorial YM-BPM, the semivectorial YM-BPM, which was not explicity described in [13], is employed in the propagating beam analysis. The semivectorial equations are expressed as follows:

$$
\begin{gathered}
-2 j \beta_{\mathrm{ref}} \frac{\partial E_{\alpha}}{\partial z}-\beta_{\mathrm{ref}}^{2} E_{\alpha}+\frac{\partial}{\partial \alpha}\left(\frac{1}{\varepsilon_{r}} \frac{\partial \varepsilon_{r} E_{\alpha}}{\partial \alpha}\right) \\
=-j k_{0}\left(\frac{\partial H_{\tilde{\alpha}}}{\partial z}-j \beta_{\mathrm{ref}} H_{\tilde{\alpha}}\right) \\
-2 j \beta_{\mathrm{ref}} \frac{\partial H_{\tilde{\alpha}}}{\partial z}-\beta_{\mathrm{ref}}^{2} H_{\tilde{\alpha}}+\frac{\partial^{2} H_{\tilde{\alpha}}}{\partial \beta^{2}} \\
=-j k_{0}\left(\varepsilon_{r} \frac{\partial E_{\alpha}}{\partial z}-j \beta_{\mathrm{ref}} \varepsilon_{r} E_{\alpha}\right)
\end{gathered}
$$

where $k_{0}$ is the free-space wavenumber, $\varepsilon_{r}\left(=n_{r}^{2}\right)$ is the relative permittivity, and $\beta_{\text {ref }}\left(=k_{0} n_{0}\right)$ is the reference propagation constant. Subscripts $\alpha$ and $\tilde{\alpha}$ are taken to be $\alpha=x$ and $\tilde{\alpha}=y$, respectively, for the quasi-TE mode and $\alpha=y$ and $\tilde{\alpha}=x$, respectively, for the quasi-TM mode.

The finite-difference equations are obtained by discretizing (1) and (2) with the use of Yee's mesh and splitting the propagation axis into two steps. Following the procedure that is similar to the full-vectorial YM-BPM in [13], we obtain the following steps:

Step 1) $(l \Rightarrow l+1 / 2)$ :

$$
\begin{aligned}
\left(A_{-}^{2} \varepsilon_{r a, b}-B_{+}^{2}+B_{+} \bar{\delta}_{\alpha}^{2}\right) E_{\alpha a, b}^{l+\frac{1}{2}} & \\
= & \left(A_{+} A_{-} \varepsilon_{r a, b}-B_{+} B_{-}\right) E_{\alpha a, b}^{l} \\
& \quad-\gamma\left(A_{-} B_{-}-A_{+} B_{+}+A_{-} \delta_{\tilde{\alpha}}^{2}\right) H_{\tilde{\alpha} a, b}^{l} \\
H_{\tilde{\alpha} a, b}^{l+\frac{1}{2}}= & \frac{1}{B_{+}}\left\{\left(B_{-}+\delta_{\tilde{\alpha}}^{2}\right) H_{\tilde{\alpha} a, b}^{l}\right. \\
& \left.+\gamma A_{-} \varepsilon_{r a, b} E_{\alpha a, b}^{l+\frac{1}{2}}-\gamma A_{+} \varepsilon_{r a, b} E_{\alpha a, b}^{l}\right\} .
\end{aligned}
$$

Step 2) $(l+1 / 2 \Rightarrow l+1)$ :

$$
\begin{aligned}
& \left(A_{-}^{2} \varepsilon_{r a, b}-B_{+}^{2}+B_{+} \delta_{\tilde{\alpha}}^{2}\right) H_{\tilde{\alpha} a, b}^{l+1} \\
& =\left(A_{+} A_{-} \varepsilon_{r a, b}-B_{+} B_{-}\right) H_{\tilde{\alpha} a, b}^{l+\frac{1}{2}} \\
& \quad-\gamma\left(A_{-} B_{-}-A_{+} B_{+}+A_{-} \bar{\delta}_{\alpha}^{2}\right) \varepsilon_{r a, b} E_{\alpha a, b}^{l+\frac{1}{2}} \\
& \quad E_{\alpha a, b}^{l+1} \\
& \quad=\frac{1}{B_{+}}\left\{\left(B_{-}+\bar{\delta}_{\alpha}^{2}\right) E_{\alpha a, b}^{l+\frac{1}{2}}+\gamma A_{-} H_{\tilde{\alpha} a, b}^{l+1}-\gamma A_{+} H_{\tilde{\alpha} a, b}^{l+\frac{1}{2}}\right\}
\end{aligned}
$$

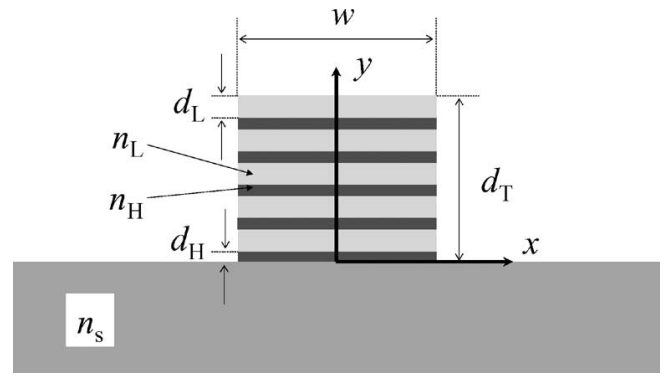

Fig. 1. Configuration of an MTFW $\left(n_{H}=2.49, n_{L}=1.47, n_{s}=1.512\right.$, and $\lambda=0.6328 \mu \mathrm{m})$.

where

$$
\begin{aligned}
A_{ \pm} & =\frac{j k_{0}\left(1 \pm \frac{j \beta_{\mathrm{ref}} \Delta z / 2}{2}\right)}{\Delta z / 2} \\
B_{ \pm} & =\frac{2 j \beta_{\mathrm{ref}} \pm \frac{\beta_{\mathrm{ref}}^{2} \Delta z / 2}{2}}{\Delta z / 2}
\end{aligned}
$$

and $\delta_{\alpha}^{2}, \delta_{\tilde{\alpha}}^{2}$, and $\bar{\delta}_{\alpha}^{2}$ are the difference operators. The coefficient $\gamma$ and subscripts $a$ and $b$ are written as

$$
\gamma=1 \quad a=i+\frac{1}{2} \quad b=j
$$

for the quasi-TE mode and

$$
\gamma=-1 \quad a=i \quad b=j+\frac{1}{2}
$$

for the quasi-TM mode.

In the present method, the preceding two-step equations are solved, updating the solution in the $z$ direction. Efficient computation can be carried out by the Thomas algorithm since (3) and (5) are the tridiagonal systems of linear equations, while (4) and (6) are explicitly solved. Eventually, the calculation is performed with the following process:

$$
(3) \rightarrow(4) \rightarrow(5) \rightarrow(6)
$$

\section{RESULT}

\section{A. Eigenmode Analysis}

In order to demonstrate the validity of this analysis, we first analyze the eigenmodes of the MTFW (Fig. 1) treated in [4]. The configuration parameters are taken as follows: The refractive indexes are $n_{H}=2.49, n_{L}=1.47$, and $n_{s}=1.512$. The thicknesses of the MTFW are $d_{H}=0.02 \mu \mathrm{m}$ and $d_{L}=$ $0.1 \mu \mathrm{m}$, resulting in a total thickness of $d_{T}=0.6 \mu \mathrm{m}$ (the total number of layers is ten). The operating wavelength is chosen to be $0.6328 \mu \mathrm{m}$ throughout this paper. The computational parameters are fixed at $\Delta x=0.025 \mu \mathrm{m}$ and $\Delta y=0.004 \mu \mathrm{m}$.

Fig. 2 shows the effective indexes as a function of waveguide width $w$. In Fig. 2, the black dots show the results of the YM-BPM, and the solid lines show the results of [4]. In the imaginary-distance procedure, the $E_{11}^{x}$ mode with the largest propagation constant is first obtained. Next, the $E_{21}^{x}$ mode is 


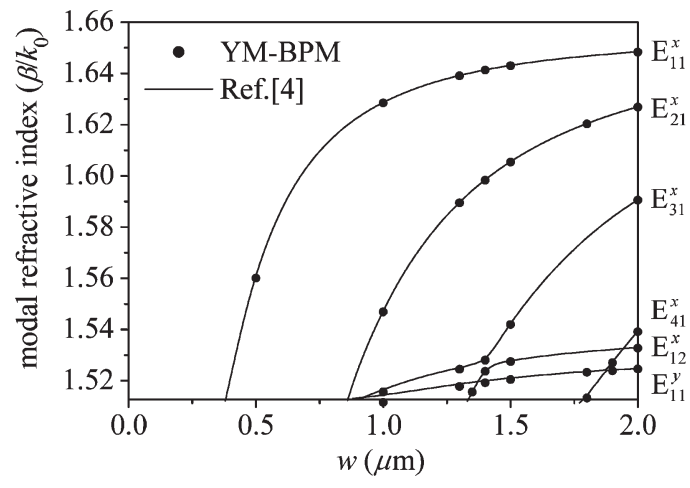

Fig. 2. Effective indexes as a function of $w$.

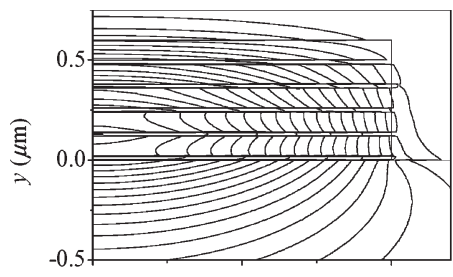

(a)

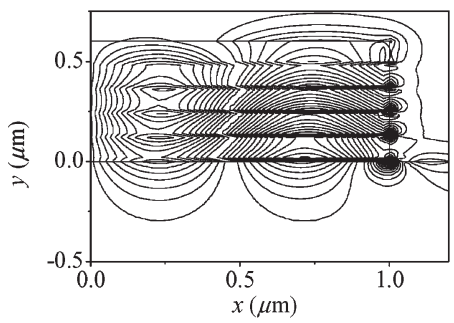

(c)

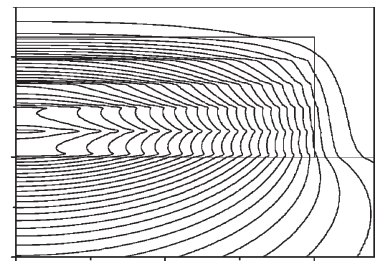

(b)

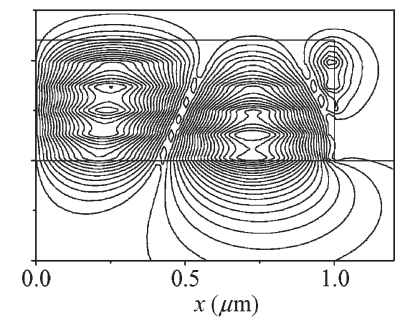

(d)
Fig. 3. Field distributions of the $E_{11}^{y}$ mode for $w=2.0 \mu \mathrm{m}$ (half region). (a) Major component of electric field $E_{y}$. (b) Major component of magnetic field $H_{x}$. (c) Minor component of electric field $E_{x}$. (d) Minor component of magnetic field $H_{y}$.

generated by subtracting the obtained $E_{11}^{x}$ mode. Moreover, the higher order modes are generated by subtracting the obtained modes. In Fig. 2, the results of the YM-BPM are in good agreement with the results of [4], including the mode-coupling regions (between the $E_{31}^{x}$ and the $E_{12}^{x}$ modes around $w=$ $1.4 \mu \mathrm{m}$ and between the $E_{41}^{x}$ and $E_{11}^{y}$ modes around $w=$ $1.8 \mu \mathrm{m})$. It is observed that the large mode separation is realized since the effective index of the $E_{11}^{x}$ mode differs significantly from that of the $E_{11}^{y}$ mode. As an example, the transverse field distributions of the $E_{11}^{y}$ mode that were observed at $w=$ $2.0 \mu \mathrm{m}$ are illustrated in Fig. 3, where only half the region is shown due to the symmetry with respect to the $y$-axis. The field discontinuities across the interfaces are clearly observed.

Consideration is next given to the rib-type MTFW coupler shown in Fig. 4. The configuration parameters are taken to be the same as those of Fig. 1. The total number of layers is ten, in which the four layers compose the rib part. The waveguide width $w$ is chosen to be $1.0 \mu \mathrm{m}$ so that this structure is regarded as two parallel polarization-maintaining waveguides, which allow only the $E_{11}^{x}$ mode to propagate.

We now investigate the coupling length for the $E_{11}^{x}$ mode as a function of waveguide spacing $s$. The results are compared with those of the single-layer rib-type waveguides coupler whose re-

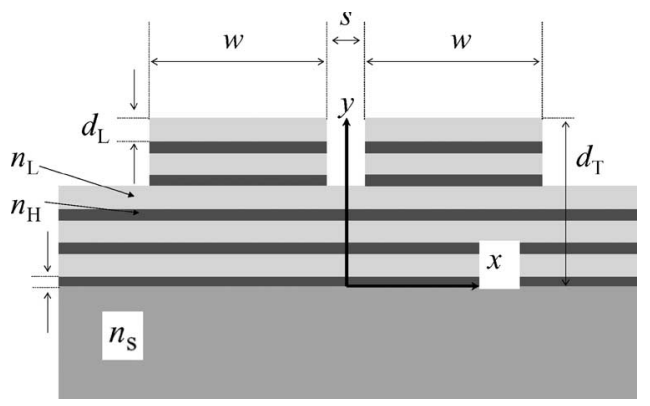

Fig. 4. Configuration of a rib-type MTFW coupler $\left(n_{H}=2.49, n_{L}=1.47\right.$, $n_{s}=1.512$, and $\left.\lambda=0.6328 \mu \mathrm{m}\right)$.

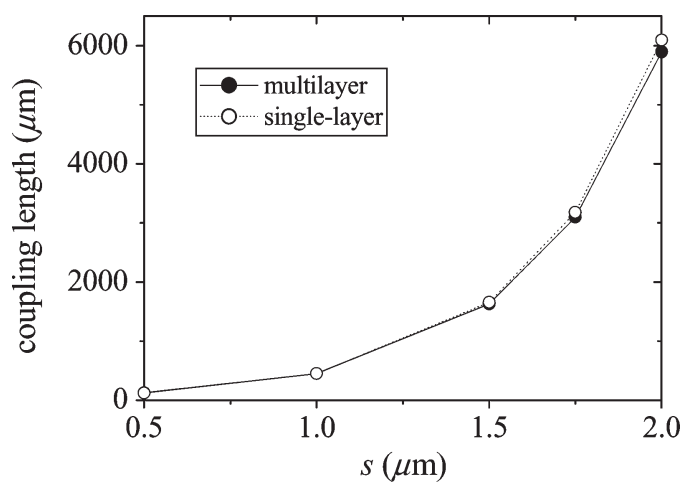

Fig. 5. Coupling length as a function of $s$.

fractive index of the guiding layer is 1.64 , which is determined from the weighted average $\left(n_{H} d_{H}+n_{L} d_{L}\right) /\left(d_{H}+d_{L}\right)$. The coupling length $L_{c}$ is calculated as

$$
L_{c}=\frac{\pi}{\beta_{\text {even }}-\beta_{\text {odd }}}
$$

where $\beta_{\text {even }}$ and $\beta_{\text {odd }}$ are the propagation constants of the even and odd supermodes, respectively. The coupling length as a function of $s$ is shown in Fig. 5. The solid and open circles show the coupling lengths of the MTFW and the single-layer waveguide, respectively. It is seen in Fig. 5 that the coupling lengths are close to each other for the $E_{11}^{x}$ mode. We should note, however, that the MTFW does not allow the $E^{y}$ modes to propagate, while the single-layer waveguide allows propagation, i.e., the MTFW can compose a polarization-maintaining coupler.

In summary, it has been demonstrated that the YM-BPM can be utilized for the eigenmode analysis of the MTFW, which yields the mode coupling. It is also shown for the $E_{11}^{x}$ mode that the coupling lengths of the rib-type MTFW coupler approximately correspond to those of the single-layer waveguide with the weighted average index.

\section{B. Directional-Coupler-Type Polarization Splitters Using MTFWs}

In this section, we propose a polarization splitter that takes advantage of the birefringence of the MTFW. For the MTFW (Fig. 1) analyzed in the previous section, the mode fields are strongly confined to the core region, resulting in a long device length. In addition, note that the rib-type MTFW (Fig. 4) 


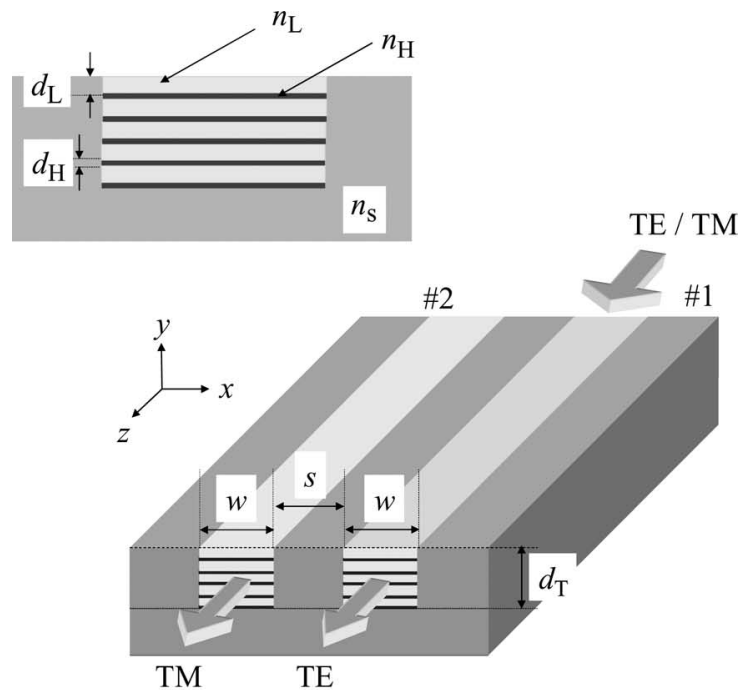

(a)

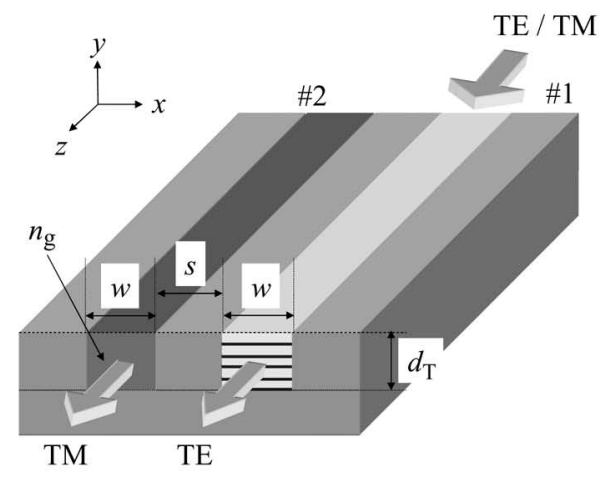

(b)

Fig. 6. Directional-coupler-type polarization splitters. (a) Type S. (b) Type A.

cannot allow the quasi-TM $\left(E^{y}\right)$ modes to propagate. We, therefore, employ an embedded-type MTFW, which contributes to realizing a short device length.

Fig. 6(a) and (b) shows the configurations of directionalcoupler-type polarization splitters. In this paper, we call the polarization splitter that is shown in Fig. 6(a) Type $S$ (symmetry), which consists of two parallel MTFWs, and another splitter that is shown in Fig. 6(b) Type A (asymmetry), whose one arm consists of the MTFW. That is, for Type A, the waveguide 2 in Type $S$ is replaced by a single-layer waveguide. Note that both types in this paper use waveguide 1 as the input waveguide, while Type A in [6] uses waveguide 2 as the input waveguide. The configuration parameters are taken to be the same as those in Fig. 1. The width of the MTFW is $w=1 \mu \mathrm{m}$, and the spacing between the two parallel waveguides is $s=1 \mu \mathrm{m}$. For Type A, the refractive index of the single-layer waveguide $n_{g}$ is taken to be variable.

First, the eigenmodes of the isolated MTFW [the upper left of Fig. 6(a)] are analyzed to demonstrate the birefringence properties of the MTFW. The field distributions of the two polarizations are shown in Fig. 7, where only half the region is illustrated. It is seen that the field confinement of the quasiTE mode differs much from that of the quasi-TM mode. For Type A in [6], the MTFW whose configuration parameters are

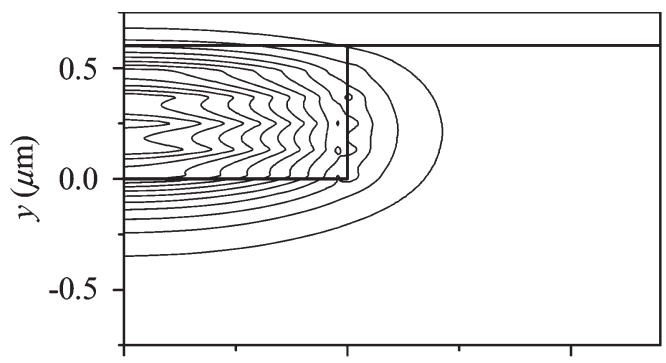

(a)

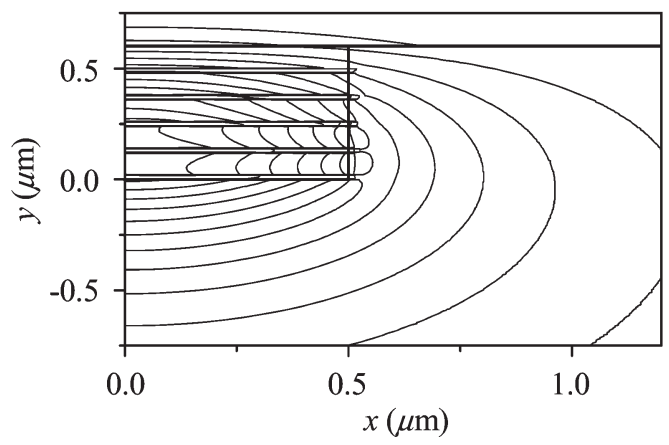

(b)

Fig. 7. Field distributions of the embedded MTFW. (a) Quasi-TE mode $\left(E_{x}\right)$. (b) Quasi-TM mode $\left(E_{y}\right)$.

TABLE I

COUPLING LENGTH

\begin{tabular}{|cc|c|c|c|}
\hline \multicolumn{2}{|c|}{ Mode } & $\beta_{\text {even }}$ & $\beta_{\text {odd }}$ & $L(\mu \mathrm{m})$ \\
\hline quasi-TE $\left(\mathrm{E}_{11}^{x}\right)$ & 16.2367 & 16.2365 & $>20000$ \\
\hline quasi-TM $\left(\mathrm{E}_{11}^{y}\right)$ & 15.0940 & 15.0826 & 277 \\
\hline
\end{tabular}

different from that of the present one was employed to cut off the quasi-TM mode in waveguide 1 , and waveguide 2 was used as the input waveguide instead of waveguide 1. Consequently, the coupling of the quasi-TE mode was utilized for splitting the polarizations. On the other hand, in this paper, we utilize the coupling of the quasi-TM mode for splitting the polarizations, leading to the advantage that the coupling length is reduced. As shown in Table I, the coupling length of the quasiTE mode is more than $20000 \mu \mathrm{m}$, and that of the quasi-TM mode is about $270 \mu \mathrm{m}$.

Next, the characteristics of the two types of polarization splitters are evaluated in the propagating beam analysis. For the polarization splitters that are considered in this paper, it is confirmed that the results that were obtained from the semivectorial BPM agree well with those from the full-vectorial BPM. Since the computational efficiency of the semivectorial BPM is higher than that of the full-vectorial BPM, the semivectorial BPM is employed in this analysis. The incident field to waveguide 1 is the eigenmode of the single MTFW that was obtained from the imaginary-distance BPM.

We show the polarization behavior for Type S. Fig. 8 shows the field distributions for the two polarizations in the $x-z$ plane at $y=0.3 \mu \mathrm{m}$. It is observed that the two polarizations can be separated at $z \simeq 270 \mu \mathrm{m}$.

Fig. 9 shows the field distributions in the $x-y$ plane at $z=270 \mu \mathrm{m}$. For the quasi-TE mode, the field confinement is very strong, so the field propagates without coupling to the 


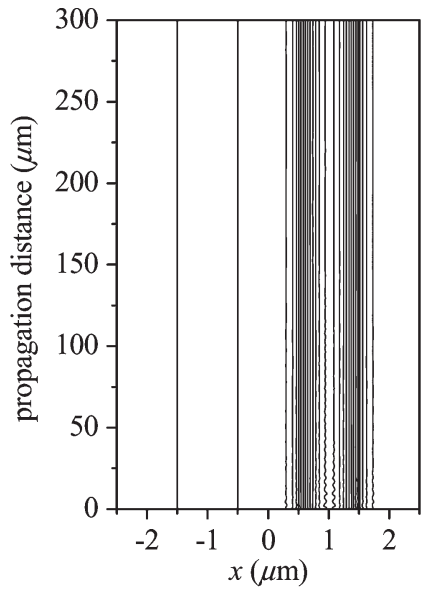

(a)

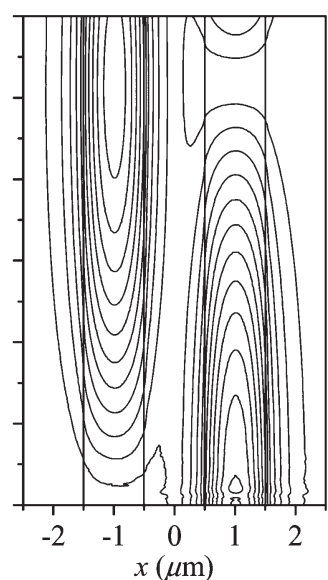

(b)
Fig. 8. Field distributions at $y=0.3 \mu \mathrm{m}$. (a) Quasi-TE mode $\left(E_{x}\right)$. (b) Quasi-TM mode $\left(E_{y}\right)$.

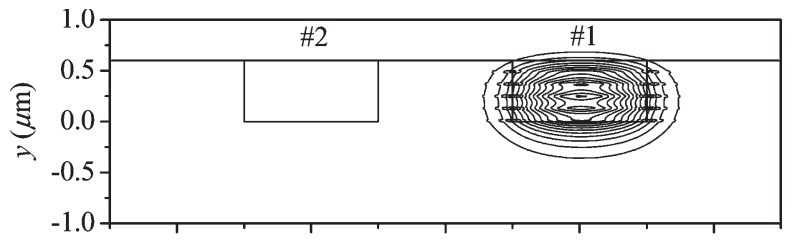

(a)

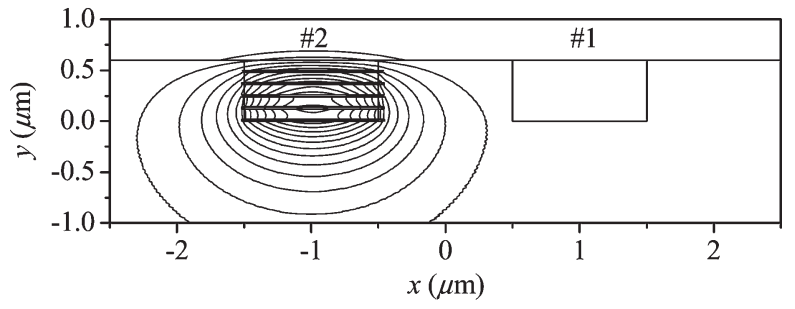

(b)

Fig. 9. Field distributions at $z=270 \mu \mathrm{m}$. (a) Quasi-TE mode $\left(E_{x}\right)$. (b) Quasi-TM mode $\left(E_{y}\right)$.

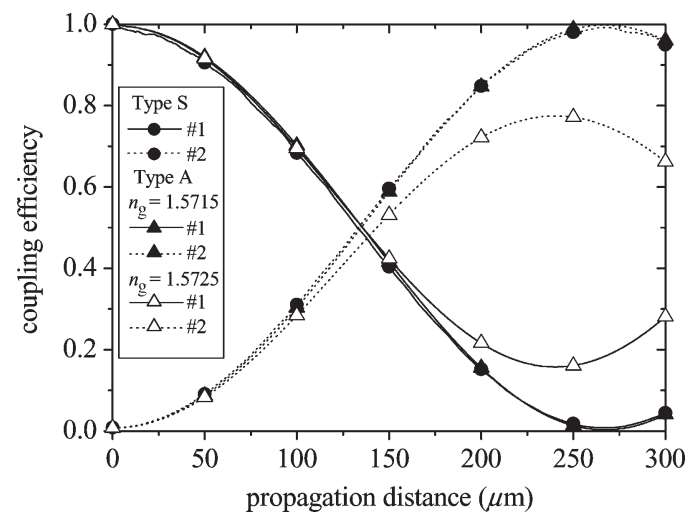

Fig. 10. Coupling efficiency.

other waveguide. In contrast, for the quasi-TM mode, the field extends into the substrate, leading to a shorter coupling length.

Fig. 10 shows the coupling efficiency for the quasi-TM mode as a function of propagation distance, in which the result for Type $S$ is shown by $\bullet$. It is observed that the coupling efficiency in waveguide 2 is more than 0.99 at $z=270 \mu \mathrm{m}$.
TABLE II

CROSSTALK

\begin{tabular}{|c|c|c|c|}
\hline Mode & Type $\mathrm{S}$ & $\begin{array}{c}\text { Type } A \\
n_{\mathrm{g}}=1.5715\end{array}$ & $\begin{array}{c}\text { Type } \AA \\
n_{\mathrm{g}}=1.5725\end{array}$ \\
\hline quasi-TE $\left(E_{11}^{x}\right)$ & $-33.5 d B$ & $-36.3 \mathrm{~dB}$ & $-36.8 d B$ \\
\hline quasi-TM $\left(\mathrm{E}_{11}^{y}\right)$ & $-20.7 \mathrm{~dB}$ & $-25.3 \mathrm{~dB}$ & $-5.9 \mathrm{~dB}$ \\
\hline
\end{tabular}

Two additional data for Type $\mathrm{A}$ are also presented to show the effects of varying the refractive index $n_{g}$ on the coupling efficiency: One is for $n_{g}=1.5715$, in which the phase-matching condition between the MTFW and the single-layer waveguide is completely satisfied, and the other is for $n_{g}=1.5725$, in which slight deviation from the phase-matching condition is included. The maximum coupling efficiency in waveguide 2 is more than 0.99 for $n_{g}=1.5715$. However, the maximum coupling efficiency is, at best, 0.8 when an error on the order of $10^{-3}$ is induced in the refractive index $n_{g}$. As a result, the fine index adjustment on the order of $10^{-4}$ is required for the refractive index of the single-layer waveguide. In contrast, the phase matching is readily satisfied for Type $S$; the requirement for fabrication accuracy is relaxed. Although not illustrated, for the quasi-TE mode, the coupling efficiency that is observed in waveguide 1 remains almost unity in any case, i.e., the light wave that is coupled into waveguide 2 is negligible.

The performance of the polarization splitter is assessed by the crosstalks (Table II) that are defined as

$$
\begin{gathered}
C R_{\mathrm{TE}}=-10 \log _{10}\left(C E_{\# 1} / C E_{\# 2}\right) \\
C R_{\mathrm{TM}}=-10 \log _{10}\left(C E_{\# 2} / C E_{\# 1}\right)
\end{gathered}
$$

where $C E_{\# 1}$ and $C E_{\# 2}$ are the coupling efficiencies at waveguides 1 and 2 , respectively. The coupling efficiency is obtained by overlapping the propagating field with the specific eigenmode field. For Type $\mathrm{S}$, the crosstalks are calculated to be $-33.5 \mathrm{~dB}$ for the quasi-TE mode and $-20.7 \mathrm{~dB}$ for the quasi-TM mode at $z=270 \mu \mathrm{m}$. The crosstalk of the quasi-TE mode is lower than that of the quasi-TM mode since the field of the quasi-TE mode is strongly confined to the core, as shown in Fig. 9. Note, however, that lower crosstalks can readily be obtained when slightly bent output waveguides are connected to the parallel waveguides. It should also be noted that, for Type A, the crosstalk is increased from -25.3 to $-5.9 \mathrm{~dB}$ when the error on the order of $10^{-3}$ is induced in the refractive index $n_{g}$.

\section{Crossing-Type Polarization Splitter Using MTFWs}

In this section, we propose an alternative polarization splitter that is composed of crossing MTFWs shown in Fig. 11. The configuration parameters of the MTFW are taken to be the same as those in Fig. 6. The overlapping length at the crossing region is $\Delta S=0.0125 \mu \mathrm{m}$. The spacing between the two waveguides at the input and output is taken to be $s=1 \mu \mathrm{m}$, which is the same as the spacing that is used in Fig. 6. The crossing angle is designated as $\theta$. The incident field to waveguide 1 is the eigenmode of the single MTFW.

To assess the performance of the mode splitter, we evaluate the coupling efficiency. Fig. 12 shows the coupling efficiency as a function of crossing angle $\theta$. We again calculate the coupling 


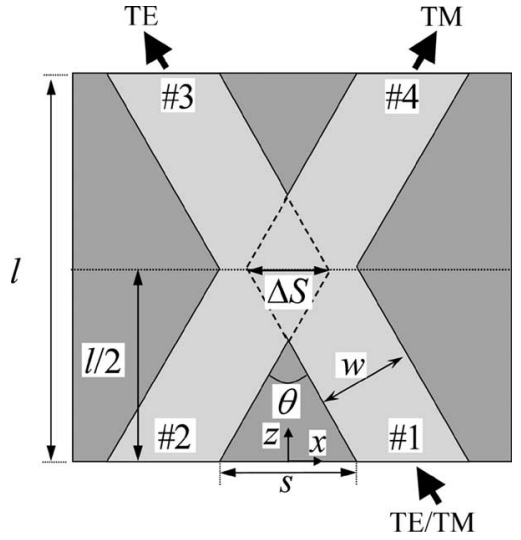

Fig. 11. Configuration of a crossing-type polarization splitter.

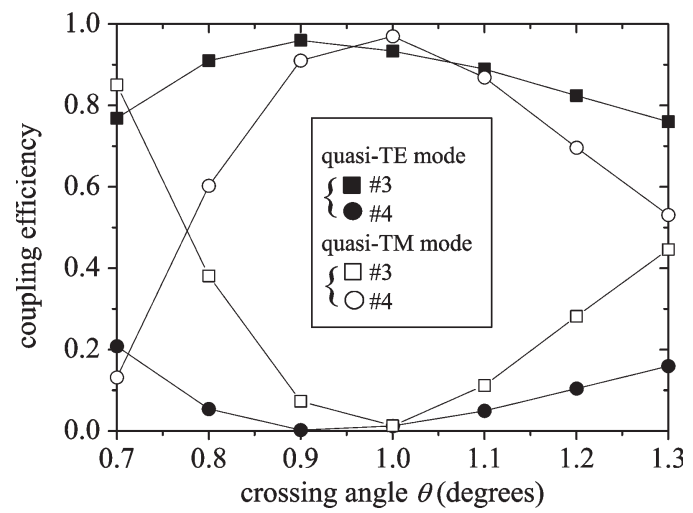

Fig. 12. Coupling efficiency as a function of crossing angle $\theta$.

efficiency using the propagating field and the eigenmode of the single MTFW. The propagating field is evaluated at $z=l$, in which $l$ is varied from 168 to $90 \mu \mathrm{m}$, as $\theta$ increases from $0.7^{\circ}$ to $1.3^{\circ}$ since $s$ is fixed to be $1 \mu \mathrm{m}$. It is found that the maximum coupling efficiency of the quasi-TE and quasiTM modes are calculated to be 0.96 at $\theta=0.9^{\circ}$ and 0.97 at $\theta=1.0^{\circ}$, respectively. However, for $\theta=0.9^{\circ}$, the coupling efficiency of undesirable polarization (the quasi-TM mode in waveguide 3 ) is higher than that for $\theta=1.0^{\circ}$. We, therefore, choose $\theta=1.0^{\circ}$, in which the crosstalks of the quasi-TE and quasi-TM modes are calculated to be -19.0 and $-19.4 \mathrm{~dB}$, respectively. (In the calculation of $C R_{\mathrm{TE}}$ and $C R_{\mathrm{TM}}, C E_{\# 1}$ and $C E_{\# 2}$ are changed to $C E_{\# 3}$ and $C E_{\# 4}$, respectively, according to the definition in Fig. 11.) It should be noted that the device length is about $l=120 \mu \mathrm{m}$, which is further reduced from that of the directional-coupler-type splitter.

\section{CONCLUSION}

The MTFWs have been analyzed using the 3-D YM-BPM. First, the eigenmodes of the MTFW are evaluated using the imaginary-distance YM-BPM. It is shown that the obtained effective indexes are in good agreement with previously published data. In the eigenmode analysis of the rib-type MTFW coupler, the coupling lengths are calculated and compared with those of the single-layer waveguide. It is found, for the $E_{11}^{x}$ mode, that the coupling length of the MTFW is approximately equal to that of the single-layer waveguide with the weighted average index of the indexes of the MTFW. Next, we propose the directional-coupler-type polarization splitter using MTFWs. The polarization splitter, which consists of the two parallel MTFWs, has the advantage that a short coupling length is obtainable. The crosstalks are calculated to be $-33.5 \mathrm{~dB}$ for the quasi-TE mode and $-20.7 \mathrm{~dB}$ for the quasi-TM mode with a coupling length of $270 \mu \mathrm{m}$. Finally, a crossing-type polarization splitter composed of MTFWs has been analyzed. The polarization splitter, which consists of the crossing MTFWs, leads to a further reduction in the device length. The crosstalks of the quasi-TE and quasi-TM modes are calculated to be about $-19 \mathrm{~dB}$ with a device length of $120 \mu \mathrm{m}$.

\section{ACKNOWLEDGMENT}

The authors would like to thank Prof. T. Anada of Kanagawa University for sending data [4] regarding the effective indexes of Fig. 2.

\section{REFERENCES}

[1] M. Born and E. Wolf, Principles of Optics. Oxford, U.K.: Pergamon, 1975, ch. XIV.

[2] M. Takeda, "Birefringence in a dielectric with periodic structure," J. Lightw. Technol., vol. LT-2, no. 4, pp. 522-527, Aug. 1984.

[3] A. Yariv and P. Yeh, "Electromagnetic propagation in periodic stratified media-Part II: Birefringence, phase matching, and X-ray lasers," J. Opt. Soc. Amer, vol. 67, no. 4, pp. 438-448, Apr. 1977.

[4] J.-P. Hsu, T. Anada, T. Hiraoka, T. M. Benson, and P. Sewell, "Method for calculating the properties of polarization maintaining $3 \mathrm{D}$ waveguides in very thin multilayered structures," Proc. Inst. Electr. Eng.-Optoelectron., vol. 146, no. 3, pp. 165-171, Jun. 1999.

[5] K. Matsubara and H. Yajima, "Y-branch TE-TM mode splitter using multilayered waveguide," Jpn. J. Appl. Phys., vol. 31, no. 5B, pp. 16361640, May 1992.

[6] K. Matsubara and H. Yajima, "Directional-coupler type TE-TM mode splitter using dielectric multilayered waveguide," IEICE Trans., vol. J77C-I, no. 10, pp. 572-575, Oct. 1994.

[7] M. Koshiba, Optical Waveguide Analysis. New York: McGraw-Hill, 1990.

[8] D. Yevick and W. Bardyszewski, "Correspondence of variation finitedifference (relaxation) and imaginary-distance propagation methods for modal analysis," Opt. Lett., vol. 17, no. 5, pp. 329-330, Mar. 1992.

[9] C. Vassallo, "1993-1995 Optical mode solvers," Opt. Quantum Electron., vol. 29, no. 2, pp. 95-114, Feb. 1997.

[10] S. M. Lee, "Finite-difference vectorial-beam-propagation method using Yee's discretization scheme for modal fields," J. Opt. Soc. Amer. A, Opt. Image Sci., vol. 13, no. 7, pp. 1369-1377, Jul. 1996.

[11] J. Yamauchi, N. Morohashi, and H. Nakano, "Rib waveguide analysis by the imaginary-distance beam-propagation method based on Yee's mesh," Opt. Quantum Electron., vol. 30, no. 5/6, pp. 397-401, 1998.

[12] T. Ando, H. Nakayama, S. Numata, J. Yamauchi, and H. Nakano, "Eigenmode analysis of optical waveguides by a Yee-mesh-based imaginarydistance propagation method for an arbitrary dielectric interface," J. Lightw. Technol., vol. 20, no. 8, pp. 1627-1634, Aug. 2002.

[13] J. Yamauchi, T. Mugita, and H. Nakano, "Implicit Yee-mesh-based finitedifference full-vectorial beam-propagation method," J. Lightw. Technol., vol. 23, no. 5, pp. 1947-1955, May 2005.

[14] A. Taflove and S. C. Hagness, Computational Electrodynamics, The Finite-Difference Time-Domain Method, 2nd ed. Norwood, MA: Artech House, 2000.

[15] J. Yamauchi, Y. Kamei, and H. Nakano, "Directional-coupler-type polarization splitters using multilayer thin-film waveguides," in OSA Integr. Photon. Res. Tech. Dig., 2004. IFC2.

[16] J. Yamauchi, K. Murase, and H. Nakano, "Crossing-type TE/TM mode splitter using a multilayer thin-film waveguide," in Proc. Contemp. Photon. Technol., Jan. 2006, pp. 94-95.

[17] J. C. Chen and S. Jüngling, "Computation of higher-order waveguide modes by imaginary-distance beam propagation method," Opt. Quantum Electron., vol. 26, no. 3, pp. S199-S205, Mar. 1994. 


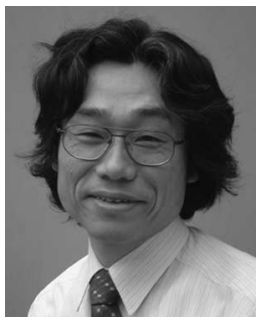

Junji Yamauchi (M'84) was born in Nagoya, Japan, on August 23, 1953. He received the B.E., M.E., and Dr.E. degrees from Hosei University, Tokyo, Japan, in 1976,1978 , and 1982 , respectively.

From 1984 to 1988, he was a Lecturer with the Department of Electrical Engineering, Tokyo Metropolitan Technical College. Since 1988, he has been a member of the Faculty of Engineering, Hosei University, where he is currently a Professor of electronic informatics. He is the author of Propagating Beam Analysis of Optical Waveguides (Baldock, Hertfordshire, U.K.: Research Studies, 2003). His research interests include optical waveguides and circularly polarized antennas.

Dr. Yamauchi is a member of the Optical Society of America and the Institute of Electronics, Information, and Communication Engineers of Japan.

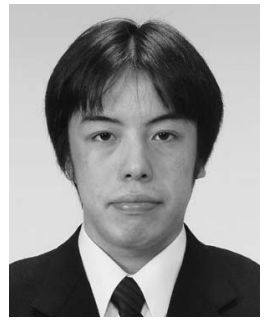

Yuji Kamei was born in Kanagawa, Japan, on November 5, 1980. He received the B.E. and M.E. degrees from Hosei University, Tokyo, Japan, in 2003 and 2005, respectively.

In 2005, he joined Topcon Corporation, Tokyo. His research interests include the numerical analysis of dielectric waveguides.

Mr. Kamei is a member of the Institute of Electronics, Information, and Communication Engineers of Japan.

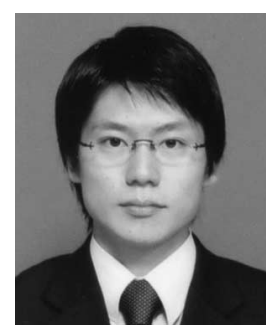

Koichi Murase was born in Tokyo, Japan, on October 15, 1982. He received the B.E. and M.E. degrees from Hosei University, Tokyo, in 2005 and 2007, respectively.

In 2007, he joined Tokyo Electric Power Company, Tokyo. His research interests include the numerical analysis of dielectric waveguides.

Mr. Murase is a member of the Institute of Electronics, Information, and Communication Engineers of Japan.

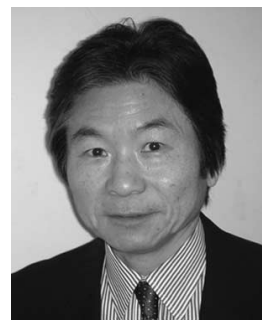

Hisamatsu Nakano (M'75-SM'87-F'92) was born in Ibaraki, Japan, on April 13, 1945. He received the B.E., M.E., and Dr.E. degrees in electrical engineering from Hosei University, Tokyo, Japan, in 1968, 1970, and 1974, respectively.

Since 1973, he has been a member of the Faculty of Engineering, Hosei University, where he is currently a Professor of electronic informatics. $\mathrm{He}$ has published more than 200 refereed journal papers and 200 international symposium papers on antenna and relevant problems. He was a Visiting Associate Professor with Syracuse University, Syracuse, NY, during May-September 1981; a Visiting Professor with the University of Manitoba, Winnipeg, MB, Canada, during March-September 1986; and a Visiting Professor with the University of California, Los Angeles, during September 1986-March 1987. $\mathrm{He}$ is the author of Helical and Spiral Antennas (New York: Research Studies, Wiley, 1987) and the chapter "Antenna analysis using integral equations," in Analysis Methods of Electromagnetic Wave Problems, Vol. 2 (Norwood, MA: Artech House, 1996). His research interests include numerical methods for antennas, electromagnetic wave scattering problems, and light wave problems.

Dr. Nakano is an Associate Editor of the IEEE Antennas and Propagation Magazine. He was the recipient of an International Scientific Exchange Award from the Natural Sciences and Engineering Research Council of Canada, the Best Paper Award from the IEE Fifth International Conference on Antennas and Propagation in 1987, the IEEE AP-S Best Application Paper Award (H.A. Wheeler Award) in 1994, and the IEEE AP-S Chen-To Tai Distinguished Educator Award in 2006. 